\title{
Utilisation de la fluorescence pour visualiser la réponse au traitement à l'iloprost des gelures
}

\author{
Mira MacLennan MD, Alexander Poole MD, Josianne Gauthier MScPharm
}

Citation : CMAJ 2021 August 9;193:E1219. doi : 10.1503/cmaj.202258-f

Voir la version anglaise de l'article ici : www.cmaj.ca/lookup/doi/10.1503/cmaj.202258

$\mathbf{U}$

n homme de 48 ans a consulté au service d'urgence de Whitehorse, au Yukon, après avoir été exposé pendant 8 heures à une température de $-47^{\circ} \mathrm{C}$. C'est un non-fumeur par ailleurs en bonne santé. Il présentait une gelure au deuxième degré au gros orteil gauche, avec cyanose jusqu'à l'articulation interphalangienne distale (annexe 1, accessible en anglais au www.cmaj.ca/ lookup/doi/10.1503/cmaj.202258/tab -related-content). Nous avons utilisé la fluorescence par injection intraveineuse de vert d'indocyanine, un colorant hydrosoluble, pour faire une première évaluation microvasculaire visuelle de la réponse au traitement. Nous avons commencé l'administration d'iloprost à une concentration de $0,2 \mu \mathrm{g} / \mathrm{mL}$, à un débit d'abord de $10 \mathrm{~mL} / \mathrm{h}$, puis de $50 \mathrm{~mL} / \mathrm{h}$ pendant 6 heures par jour, sur une période de 5 jours. Nous avons capté des images en fluorescence avant le traitement à l'iloprost (figure 1A) et immédiatement après la première perfusion de 6 heures (figure 1B), et observé une amélioration de la perfusion microvasculaire de l'orteil affecté. Le patient a bien toléré le traitement et n'a pas eu besoin d'amputation.

On dispose de peu de données épidémiologiques sur les gelures. Une étude finlandaise a fait état d'une incidence de 2,5 cas par 100000 personnes dans le pays ${ }^{1}$. Le sexe masculin, l'âge avancé et les températures de $-20^{\circ} \mathrm{C}$ ont été associés à un risque accru ${ }^{1}$. Les gelures varient en gravité entre le premier degré (légère) et le quatrième degré (grave, forte probabilité d'amputation), et peuvent causer une morbidité substantielle sous forme de sensibilité, de raideur
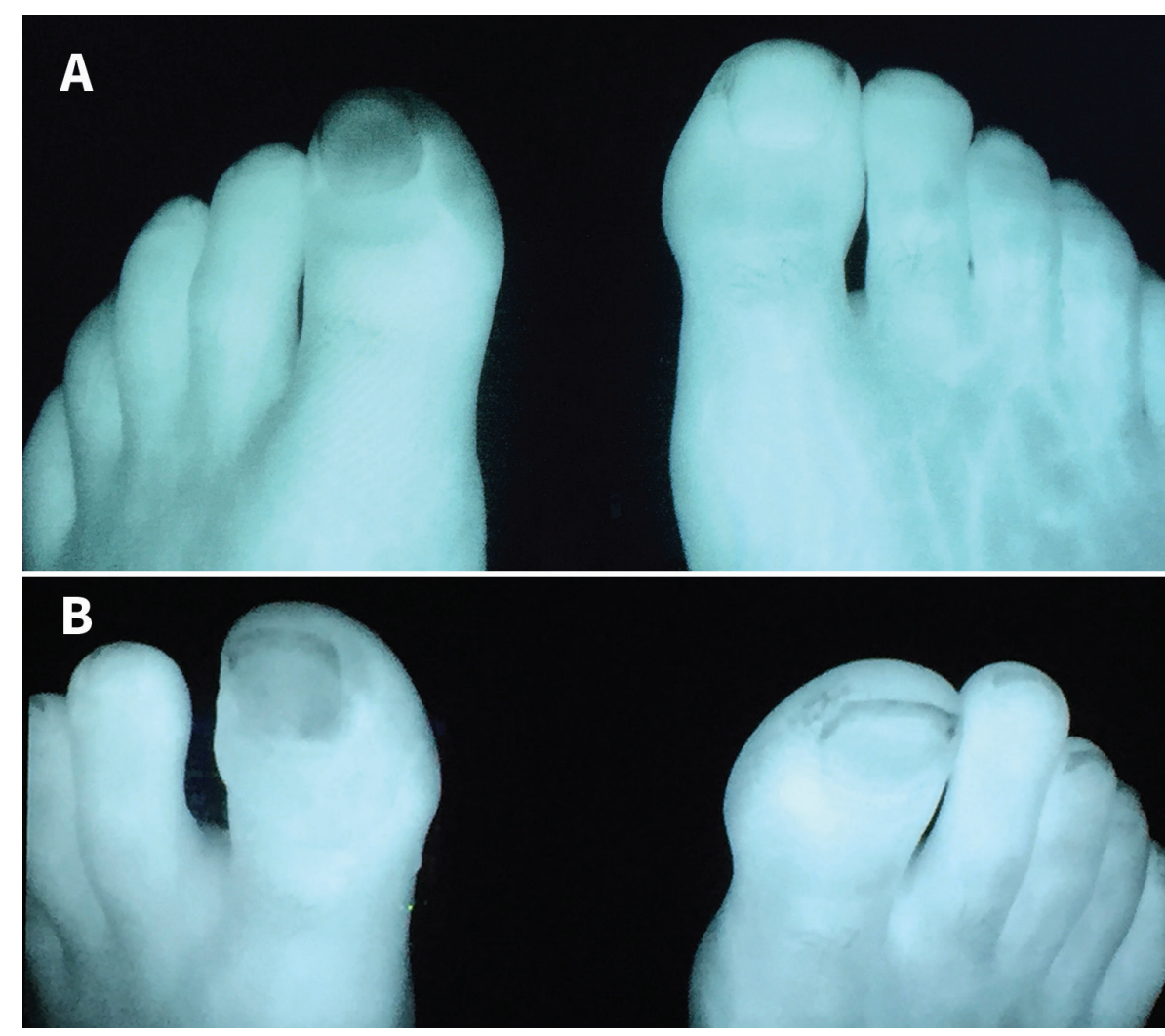

Figure 1 : Évaluation microvasculaire par injection intraveineuse de vert d'indocyanine d'une gelure au pied gauche d'un homme de 48 ans. A) 12 heures après la gelure, et avant l'administration d'iloprost : trouble circulatoire visible à la première phalange distale gauche, comparativement à la première phalange droite, qui est intacte. B) 6 heures après la fin de la première perfusion d'iloprost : amélioration de la circulation sanguine à la première phalange gauche.

articulaire et de douleur neuropathique ${ }^{2}$. Il n'existe pas de consensus sur les normes de traitement des gelures, mais de nombreux centres européens utilisent l'iloprost pour les gelures au deuxième, troisième et quatrième degré, et certains utilisent aussi l'altéplase pour les lésions au quatrième degrés ${ }^{3,4}$. Notre établissement a mis au point un protocole de traitement des gelures qui comprend des perfusions quotidiennes d'iloprost pendant 5 jours pour les lésions au deuxième, troisième et quatrième degré ses $^{\text {. Letients }}$ 
ambulatoires qui tolèrent la première perfusion peuvent recevoir les suivantes en clinique externe.

L'injection par intraveineuse de vert d'indocyanine pourrait être un moyen critique de caractériser la réponse des patients au traitement des gelures et d'optimiser les soins ${ }^{6}$. La perfusion microvasculaire est évaluée visuellement par la présence ou l'absence de vert d'indocyanine, avec une lumière dans le proche infrarouge et un moniteur. La comparaison de l'intensité de la coloration avec les tissus avoisinants permet une évaluation subjective de la perfusion relative. Les effets indésirables sont surtout liés à des réactions allergiques, et la seule contre-indication semble être l'allergie à l'iode ${ }^{7}$.

\section{Références}

1. Juopperi K, Hassi J, Ervasti O, et al. Incidence of frostbite and ambient temperature in Finland, 1986-1995. A national study based on hospital admission. Int J Circumpolar Health 2002;61:352-62.

2. Handford C, Buxton P, Russell K, et al. Frostbite: a practical approach to hospital management. Extrem Physiol Med 2014;3:7.

3. Cauchy E, Cheguillaume B, Chetaille E. A controlled trial of prostacyclin and rt-PA in the treatment of severe frostbite. N Engl J Med 2011;364:189-90.

4. Cauchy $E$, Davis CB, Pasquier M, et al. A new proposal for management of severe frostbite in the austere environment. Wilderness Environ Med 2016;27:92-9.

5. Poole A, Gauthier J. Treatment of severe frostbite with iloprost in northern Canada. CMAJ 2016;188:1255-8.

6. Masters T, Omodt S, Gayken J, et al. Microangiography to monitor treatment outcomes following severe frostbite injury to the hands. J Burn Care Res 2018;39:162-7.

7. Alander JT, Kaartinen I, Laakso A, et al. A review of indocyanine green fluorescent imaging in surgery. Int J Biomed Imaging 2012;2012: 940585.

Intérêts concurrents : Aucun déclaré.

Cet article a été révisé par des pairs.

Les auteurs ont obtenu le consentement du patient.

Affiliations : Département de chirurgie (MacLennan), Université McMaster, Hamilton, Ont.; École de santé publique T. H. Chan de l'Université Harvard (MacLennan), Boston (Massachusetts); Hôpital général de Whitehorse (Poole, Gauthier), Whitehorse, Yn.; Département de chirurgie (Poole), Faculté de médecine Cumming, Université de Calgary, Calgary, Alb.; Université de la Saskatchewan (Gauthier), Saskatoon, Sask.

Propriété intellectuelle du contenu : Il s'agit d'un article en libre accès distribué conformément aux modalités de la licence Creative Commons Attribution (CC BY-NC-ND 4.0), qui permet l'utilisation, la diffusion et la reproduction dans tout médium à la condition que la publication originale soit adéquatement citée, que l'utilisation se fasse à des fins non commerciales (c.-à-d., recherche ou éducation) et qu'aucune modification ni adaptation n'y soit apportée. Voir : https://creativecommons.org/licenses/ by-nc-nd/4.0/deed.fr.

Correspondance : Mira MacLennan, mira.maclennan@medportal.ca 